\title{
Evolution of the Clinical Microbiology Laboratory
}

Copyright 2020 by . 


\begin{abstract}
Over the course of nearly 150 years, the clinical laboratory has diagnosed infectious diseases and identified their causative agents using a variety of approaches. These approaches can be broadly placed into three categories: biochemical, or growth-based methods, molecular and genomic diagnostics, and biomarker and serological detection of blood components. The principle of the biochemical approach is based on isolating an unknown microorganism before conducting a series of growth-based and preformed enzyme detection tests to determine an identification and subsequent antimicrobial susceptibilities. The molecular approach is the newest diagnostic approach utilized by the laboratory and is based on detection of the genetic components of an unknown organism, either isolated or directly in a clinical specimen. There are a variety of molecular techniques, with the polymerase chain reaction serving as the bases of most currently available methods. The detection of infection and inflammatory indicators, as well as serological molecules, have been utilized for diagnostic purposes nearly as long as growth-based identification methods. The biomarker approach to infectious disease diagnosis has primarily occurred outside of the traditional microbiology department, usually within chemistry and hematology where large scale automated instruments provide rapid results. With this focus series we present a concise review, with a brief history, of these different approaches by describing their underlying methodology and advantages and disadvantages, while highlighting specific examples of each, and the internal and external factors which influenced their development.
\end{abstract}




\section{Abbreviations}

FDA - Food and Drug Administration, MALDI-TOF - Matrix Assisted Laser Desorption Ionized-Time of Flight, MS - Mass Spectrometry, PCR - Polymerase Chain Reaction 


\section{Index Terms}

Biochemical, biomarkers, diagnostic microbiology, molecular testing 


\section{Learning Outcomes}

1. List the three different diagnostic approaches to infectious disease and organism identification.

2. Briefly describe each of the diagnostic approaches and identify a specific example of each one.

3. Recognize emergent biomarkers for the detection of bacterial and fungal infections, and state the clinical utility of each. 
Infectious diseases have plagued humanity for millennia, yet it was only 140 years ago that the link between infection and microorganism was established. Since that time, various laboratory approaches and techniques have been developed and deployed to increase sensitivity and specificity in the identification of infectious agents. These approaches can be categorized into three separate areas based on identification: 1) biochemical, or culture-based techniques requiring organism isolation, 2) molecular and genomic methods, and 3) detection of infectious disease indicators and microbial markers detected in blood, including serology (that are often analyzed outside the microbiology department in the "core" laboratory).

The biochemical, culture-based approach was the initial identification method established, in the form of plated and tubed media containing various growth substrates, such as carbohydrates and proteins. ${ }^{1,2}$ Later, it included the use of rapid (spot) enzyme testing, ${ }^{3,4}$ further establishing the biochemical methodology as an accurate, adaptable, and versatile form of routine bacterial identification. By the 1950s, in the face of increasing population, healthcare demand, and private and public insurance availability, ${ }^{5,6}$ the first significant advancement of the biochemical approach came in the form of miniaturized multi-test media and kits, ${ }^{7-9}$ which simply took the existing principle of identification and shrunk it. This allowed for reduced labor, media, and waste disposal costs, while providing accurate identification in a timely manner. ${ }^{10}$ The advantages of the miniaturized kit approach were further realized with the incorporation of automated, growth-based instrumentation, ${ }^{11-13}$ which remains the standard for bulk identification of bacteria and many yeast today. ${ }^{14,15}$

Only within the last $10-15$ years have we seen the basic biochemical principle of growth dependency being replaced as the method for routine organism identification. Mass spectrometry, an analytic chemistry technique used for decades, has been adapted for use as a 
powerful tool for the diagnostic microbiology lab. ${ }^{16,17}$ Matrix-assisted laser desorption ionization time-of-flight mass spectrometry (MALDI-TOF MS), as it is specifically known in the diagnostic laboratory, produces a unique protein fragment "fingerprint" for many different microorganisms, bacterial and fungal alike. ${ }^{18,19}$ The advantages of MALDI-TOF cannot be overstated; it provides the most accurate and cost effective identification in the shortest amount of time of any technique, once an organism has been isolated. ${ }^{20-22}$ Of course, there are some limitations of current mass spectrometry methods, chief among them the initial cost of the instrument and the need to consistently update the proprietary databases. ${ }^{23,24}$ However, the initial cost aside, mass spectrometry is likely to become more common as laboratories continue to look for ways to handle increasing test volume while providing the fastest turn-around-time possible. Molecular diagnostics made its debut as polymerase chain reaction (PCR)-based testing seeking to identify single, specific organisms from patient samples. ${ }^{25}$ The area of the microbiology laboratory that benefitted most from these early tests was the virology section, as virology testing typically required mammalian cultures to propagate the virus and can take up to two weeks before identification was possible. ${ }^{26}$ Since this first implementation of molecular diagnostics, new testing systems and modifications to PCR technologies have come about, reducing hands-on time with test setup, while decreasing costs and the time to result, as well as enhancing the sensitivity and specificity toward the target organism. ${ }^{27}$ An example of a current advancement that is being implemented in the microbiology laboratory is syndromic panel testing, which may concomitantly detect several common infectious organisms associated with particular body system afflictions, such as the upper respiratory and gastrointestinal tract. ${ }^{28}$

Traditional culture-based identification methods are still a cornerstone of clinical laboratory testing and will most likely remain so in the near future, however, molecular-based 
testing is becoming increasingly prevalent in microbiology laboratories. This is evident by the increasing number of molecular tests receiving and seeking FDA approval for clinical use, ${ }^{29}$ including next generation sequencing and whole genome sequencing of organisms found in patient samples. ${ }^{30}$ With this potentially forthcoming paradigm shift in organism identification comes numerous advantages and caveats that must be thoroughly considered before implementing any of these systems. These considerations will need to be assessed based on an individual laboratory's needs as there is not yet a "one size fits all" scheme for implementation. Nevertheless, molecular testing will continue to improve and remain a staple in microorganism identification and diagnostics.

Parallel to the biochemical, culture-based and molecular technological developments in the microbiology laboratory, there is a quest to find biomarkers with increased sensitivity and specificity for early identification of infection, especially for diagnosis of sepsis. Traditional laboratory markers, such as the white blood cell count and erythrocyte sedimentation rate are longstanding biomarkers of infection and inflammation. ${ }^{31,32}$ Research efforts to identify emergent biomarkers for sepsis led to the inclusion of lactate and procalcitonin as effective biomarkers. ${ }^{33}$ Several promising emergent biomarkers are being studied for sepsis, including pentraxin 3 and presepsin, which offer quicker diagnosis and prognosis.

Biomarker research expands beyond sepsis and includes promising emergent biomarkers for other bacterial and fungal infections. For example, the human neutrophil elastase and cathepsin $\mathrm{G}$ are being investigated as biomarkers for chronic wound infections as early detection can prevent progression to systemic infection. ${ }^{34,35}$ For bacterial pneumonia, timely treatment is critical and interleukin-6, a cytokine in the acute phase response, is being studied as a potential biomarker. ${ }^{36}$ Currently, detection of galactomannan allows for rapid testing of infections caused 
by pathogenic species of Aspergillus, while other potential biomarkers for invasive fungal infections are being investigated. ${ }^{37}$

The technological advances seen throughout the history of diagnostic microbiology were spurred on by the necessity of immediate, quality healthcare service for a growing population and this will continue to factor in future developments. Yet, each laboratory must consider additional factors unique to their situation, such as their budget, patient population of service, and volume of testing before deciding whether to implement the newest molecular syndromic testing panel, replace their current automated biochemical analyzer with MALDI-TOF, or validate a new biomarker for disease detection. Nevertheless, newer approaches and technology are becoming more commonly utilized, and will likely continue to do so as laboratories consolidate into fewer, but larger, facilities looking to maximize efficiency through automation.

With this focus series we present a concise review, with brief history, of these different approaches by describing their underlying methodology and advantages and disadvantages, while highlighting specific examples of each, and the internal and external factors which influenced their development. This focus series is intended to be a basic review of the topic and is written for a general clinical audience. 


\section{References}

1. Muir R. Manual of bacteriology: YJ Pentland; 1899.

2. MacConkey A. Lactose-Fermenting Bacteria in Faeces. Journal of Hygiene. 1905;5(3):333-79.

3. Ljutov V. Technique of Indole Test. Acta Pathologica et Microbiologica Scandinavica. 1959;46(4):349-60.

4. Steel K. Microbial identification. Microbiology. 1965;40(1):143-8.

5. Arestad FH. Hosptial Service in the United States: Twenty-Fifth Annual Presentation of Hospital Data by the Council on Medical Education and Hospitals of the American Medical Association. Journal of the American Medical Association. 1946;130(16):1073-161.

6. Andersen R, Hull JT. Hospital utilization and cost trends in Canada and the United States. Health services research. 1969;4(3):198-222.

7. Kohn J, editor A 2-Tube Technique for the Identification of Organisms of the Enterobacteriaceae Group. Journal of Pathology and Bacteriology; 1954: John Wiley \& Sons Ltd Baffins Lane Chichester, W Sussex P019 1UD, England.

8. Buissière J, Nardon P. Nardon P. Microméthode d'identification des bactéries. I. Intérèt de la quantification des caractèrs biochimiques. Ann Inst Pasteur (Paris). 1968;115(2):218-31.

9. Washington JA, Yu PKW, Martin WJ. Evaluation of Accuracy of Multitest Micromethod System for Identification of Enterobacteriaceae. 1971;22(3):267-9.

10. Bartlett RC, Kohan TS, Rutz C. Comparative Costs of Microbial Identification Employing Conventional and Prepackaged Commercial Systems. American Journal of Clinical Pathology. 1979;71(2):194-200.

11. Staneck JL, Vincelette J, Lamothe F, Polk EA. Evaluation of the Sensititre system for identification of Enterobacteriaceae. 1983;17(4):647-54.

12. Truant AL, Starr E, Nevel CA, Tsolakis M, Fiss EF. Comparison of AMS-Vitek, microScan, and autobac series II for the identification of gram-negative bacilli. Diagnostic Microbiology and Infectious Disease. 1989;12(3):211-5.

13. Stager CE, Davis JR. Automated systems for identification of microorganisms. 1992;5(3):302-27.

14. Eigner U, Schmid A, Wild U, Bertsch D, Fahr A-M. Analysis of the comparative workflow and performance characteristics of the VITEK 2 and Phoenix systems. Journal of clinical microbiology. 2005;43(8):3829-34.

15. Jin W-Y, Jang S-J, Lee M-J, Park G, Kim M-J, Kook J-K, et al. Evaluation of VITEK 2, MicroScan, and Phoenix for identification of clinical isolates and reference strains. Diagnostic microbiology and infectious disease. 2011;70(4):442-7.

16. Anhalt JP, Fenselau C. Identification of bacteria using mass spectrometry. Analytical Chemistry. 1975;47(2):219-25.

17. Krishnamurthy T, Ross PL. Rapid Identification of Bacteria by Direct Matrix-assisted Laser Desorption/Ionization Mass Spectrometric Analysis of Whole Cells. Rapid Communications in Mass Spectrometry. 1996;10(15):1992-6.

18. Neville SA, LeCordier A, Ziochos H, Chater MJ, Gosbell IB, Maley MW, et al. Utility of MatrixAssisted Laser Desorption Ionization-Time of Flight Mass Spectrometry following Introduction for Routine Laboratory Bacterial Identification. 2011;49(8):2980-4.

19. De Carolis E, Posteraro B, Lass-Flörl C, Vella A, Florio A, Torelli R, et al. Species identification of Aspergillus, Fusarium and Mucorales with direct surface analysis by matrix-assisted laser desorption ionization time-of-flight mass spectrometry. Clinical microbiology and infection. 2012;18(5):475-84. 
20. Seng P, Drancourt M, Gouriet F, La Scola B, Fournier P-E, Rolain JM, et al. Ongoing Revolution in Bacteriology: Routine Identification of Bacteria by Matrix-Assisted Laser Desorption Ionization Time-of-Flight Mass Spectrometry. Clinical Infectious Diseases. 2009;49(4):543-51.

21. Gaillot O, Blondiaux N, Loïez C, Wallet F, Lemaître N, Herwegh S, et al. Cost-effectiveness of switch to matrix-assisted laser desorption ionization-time of flight mass spectrometry for routine bacterial identification. Journal of clinical microbiology. 2011;49(12):4412-.

22. Tan KE, Ellis BC, Lee R, Stamper PD, Zhang SX, Carroll KC. Prospective Evaluation of a MatrixAssisted Laser Desorption Ionization-Time of Flight Mass Spectrometry System in a Hospital Clinical Microbiology Laboratory for Identification of Bacteria and Yeasts: a Bench-by-Bench Study for Assessing the Impact on Time to Identification and Cost-Effectiveness. 2012;50(10):3301-8.

23. Carroll KC, Patel R. Systems for Identification of Bacteria and Fungi. Manual of Clinical Microbiology. 12015. p. 29-43.

24. Wieser A, Schneider L, Jung J, Schubert S. MALDI-TOF MS in microbiological diagnosticsidentification of microorganisms and beyond (mini review). Applied Microbiology Biotechnology. 2012;93(3):965-74.

25. Das S, Shibib DR, Vernon MO. The new frontier of diagnostics: Molecular assays and their role in infection prevention and control. American journal of infection control. 2017;45(2):158-69.

26. Josko D. Molecular virology in the clinical laboratory. Clinical Laboratory Science. 2010;23(4):231.

27. Fairfax MR, Bluth MH, Salimnia H. Diagnostic Molecular Microbiology: A 2018 Snapshot. Clinics in laboratory medicine. 2018;38(2):253-76.

28. Ramanan P, Bryson AL, Binnicker MJ, Pritt BS, Patel R. Syndromic panel-based testing in clinical microbiology. Clinical microbiology reviews. 2018;31(1):e00024-17.

29. FDA Nucleic Acid Based Tests - Microbial Tests: U.S. Food and Drug Administration 2019 [cited 2019 July 12]. Available from: https://www.fda.gov/medical-devices/vitro-diagnostics/nucleicacid-based-tests.

30. Deurenberg RH, Bathoorn E, Chlebowicz MA, Couto N, Ferdous M, García-Cobos S, et al. Application of next generation sequencing in clinical microbiology and infection prevention. Journal of biotechnology. 2017;243:16-24.

31. Smock K, Perkins S. Examination of the blood and bone marrow. Wintrobe's Clinical Hematology2014. p. 1-64.

32. Bray C, Bell LN, Liang H, Haykal R, Kaiksow F, Mazza JJ, et al. Erythrocyte sedimentation rate and $\mathrm{C}$-reactive protein measurements and their relevance in clinical medicine. $\mathrm{Wmj}$. 2016;115(6):317-21.

33. Surviving Sepsis Campaign: Society of Critical Care Medicine; 2019 [cited 2019 May 1]. Available from: www.survivingsepsis.org.

34. Blokhuis-Arkes MH, Haalboom M, van der Palen J, Heinzle A, Sigl E, Guebitz G, et al. Rapid enzyme analysis as a diagnostic tool for wound infection: Comparison between clinical judgment, microbiological analysis, and enzyme analysis. Wound repair and regeneration. 2015;23(3):345-52.

35. Tegl G, Schiffer D, Sigl E, Heinzle A, Guebitz GM. Biomarkers for infection: enzymes, microbes, and metabolites. Applied microbiology and biotechnology. 2015;99(11):4595-614.

36. Karakioulaki M, Stolz D. Biomarkers in Pneumonia-Beyond Procalcitonin. International journal of molecular sciences. 2019;20(8):2004.

37. Ramanan P, Wengenack NL, Theel ES. Laboratory Diagnostics for Fungal Infections: A Review of Current and Future Diagnostic Assays. Clinics in chest medicine. 2017;38(3):535-54. 


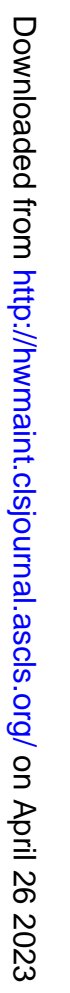

